\title{
Creative Tools for Producing Realistic 3D Facial Expressions and Animation
}

\begin{abstract}
Creative exploration of realistic 3D facial animation is a popular but very challenging task due to the high level knowledge and skills required. This forms a barrier for creative individuals who have limited technical skills but wish to explore their creativity in this area. This paper proposes a new technique that facilitates users' creative exploration by hiding the technical complexities of producing facial expressions and animation. The proposed technique draws on research from psychology, anatomy and employs Autodesk Maya as a use case by developing a creative tool, which extends Maya's Blend Shape Editor. User testing revealed that novice users in the creative media, employing the proposed tool can produce rich and realistic facial expressions that portray new interesting emotions. It reduced production time by $25 \%$ when compared to Maya and by $40 \%$ when compared to 3DS Max equivalent tools.
\end{abstract}

Keywords: computer-aided animation; creative computing; facial expression; facial animation; digital entertainment; digital creativity; usability.

\section{INTRODUCTION}

Faces play an important part in human life as they are fundamental for social interaction and the communication of emotion. Facial expression is the major form of nonverbal communication for humans. The presentation of thoughts and feelings in individual facial actions provide insight into human emotions. Emotions form the epicentre of human creativity and expression. Today's digital era is characterized by a user-led digital media renaissance that expands from taking photos and video to producing $3 \mathrm{D}$ content. This is especially true, as more and more people are exploring creative ways of generating realistic animations, particularly of human facial expressions in a computer environment. People with non-technical skills are encouraged, especially, by the popularity of 3D animation in films and video games to explore further their creative aspirations in producing their own facial expressions and animation.

Production of realistic facial animation is, however, one of the most challenging areas in animation, as any slight fault in the modelling of expression is clearly noticeable, even by inexperienced users and audiences. This is due to the human mind being tuned to facial expression perception since birth. One can see this by looking at the past 3D animated film success stories of big studios, such as Disney and Pixar, which have been with non-human characters, like animals and in-animate objects. This is because we are desensitized to imperfections in the animation due to having no experience with these characters (Waters and Levergood, 1995).

Facial animation has several creative applications beyond film and entertainment, such as virtual character performance, mobile applications and computer games. However the production of lifelike 3D human facial expressions proves a challenging and time consuming task that on one hand demands significant technical and creative expertise (Schmidt et al, 2004). On the other hand it requires complex and expensive systems (such as motion 
capture), which are operated by highly skilled and technical individuals. These technical constraints hinder creativity, resulting in the exclusion of individuals from the production and creative exploration in this area.

The aim of the research work presented here is to develop a creative product, with the focus to improving creativity and expressiveness (Hugill and Yang, 2013). That is facilitated through the development of a new technique and tool, which better adapts existing commercial animation software to serve the needs of the creative community. This is realized by making the technical requirements of the production, as much as possible, transparent to users, allowing them to focus on the creative aspects of their work. Rather than reinventing the wheel by developing new 3D tools, existing commercial professional 3D modelling and animation tools, currently used by the design community, have been employed but extended and bespoken to widen their reach to the creative and artistic communities as well.

The paper is divided into several sections. Section 2 presents the related work looking at the psychology behind the face, modelling techniques and facial animation and related software. Section 3 presents the proposed methodology and implementation and is followed by a section on the user interface that binds all together. Lastly section 4 discusses the initial results and user feedback leading into the paper conclusion and suggestions for future work.

\section{RELATED WORK}

It is only in recent years that progress made through the use new technology (3D scanners and motion capture systems) has enabled the creative exploration and incorporation of human characters in 3D animated films. 3D scanners and photometric techniques are able to digitize facial geometries and textures (Zhang et al, 2002). These techniques permit developers to take a step closer to making a character look more realistic. However this also makes people less forgiving of imperfections within the animation (Borshukov and Lewis, 2003). If the character looks like a real person, viewers would expect it to behave like one (Waters and Levergood, 1995).

The movie "Final Fantasy: the Spirits Within" (2001) was considered a milestone in the creative fusion of $3 \mathrm{D}$ characters and facial animation as it portrayed realistic looking humans in a completely computer generated movie. The film production of "Beowulf" (2007) has taken facial expression and animation creativity a step further by utilizing $3 \mathrm{D}$ scanners for the capture of the human actor's facial texture/skin as well as by employing motion capture technology for the animation of the actors' facial expressions.

Computer modelling and animation of facial expression is not a new endeavour. Initial efforts in this area began more than 30 years ago (Parke and Waters, 1996). In the 1970s, Frederic I. Parke used 3D models to develop a mesh of 3D points, which were controlled by a set of conformation and expression parameters (Parke, 1974). 


\begin{tabular}{|c|c|c|}
\hline Facial Animation Milestones & & Character Animation \\
\hline $\begin{array}{l}\text { Beowulf: a } 100 \% \text { CGI film } \\
\text { using advanced mocap } \\
\text { technology to transform live } \\
\text { action into animation. }\end{array}$ & 2001 & $\begin{array}{l}\text { Polar express: Every character } \\
\text { in the film was created using } \\
\text { facial \& body motion capture. }\end{array}$ \\
\hline $\begin{array}{l}\text { Final Fantasy: The Spirits } \\
\text { Within, citied as the first } \\
\text { computer generated movie } \\
\text { showing realistic humans. }\end{array}$ & \multirow[b]{2}{*}{1997} & $\begin{array}{l}\text { Monsters Inc, new character } \\
\text { plug in developed, for virtual } \\
\text { fur using real world physics. }\end{array}$ \\
\hline $\begin{array}{l}\text { Pixar create Geri's Game a } \\
\text { short CG film }\end{array}$ & & $\begin{array}{l}\text { Jurassic Park was the first time } \\
\text { characters with skin, muscle } \\
\text { and textures were used in a } \\
\text { film. }\end{array}$ \\
\hline
\end{tabular}

The 1990's seen increasing activities in the development of facial animation techniques and the use of computer facial animation as a key story telling component as illustrated in such films as "Toy Story" (1995).

1990

In the late 1980's Waters developed a muscle based model, Magnenat-Thalmann et al developed an abstract muscle action model, and Lewis and Hill developed approaches to automatic speech synchronisation.

\begin{tabular}{|l|l|l|}
\hline $\begin{array}{l}\text { Short animated film called } \\
\text { "Tony de Peltrie" was a } \\
\text { landmark for facial animation }\end{array}$ & $\mathbf{1 9 8 5}$ & $\begin{array}{l}\text { Tron, released by Disney was } \\
\text { the first movie to introduce } \\
\text { computer animation (CGI) }\end{array}$ \\
\hline
\end{tabular}

Platt developed the first physical based muscle controlled face model in the early 1980 's. Brennan further enhanced the development techniques of facial caricatures.

\begin{tabular}{|l|l|l|}
\hline $\begin{array}{l}\text { Parke developed a } \\
\text { parameterised three- } \\
\text { dimensional facial model }\end{array}$ & $\begin{array}{l}\text { Ekman and Friesen introduce } \\
\text { FACS (Facial Action Coding } \\
\text { System) }\end{array}$ \\
\hline $\begin{array}{l}\text { Gillenson developed an } \\
\text { interaction system to assemble } \\
\text { and edit line drawn facial } \\
\text { images. }\end{array}$ & 1973 & $\begin{array}{l}\text { Ed Catmull develops an } \\
\text { animation scripting language. } \\
\text { Using this he created an } \\
\text { animation of a smooth shaded } \\
\text { hand. }\end{array}$ \\
\hline First 3D animation by Parke & 1970 & \\
\hline
\end{tabular}

Fig. 1. Facial Character Animation Key Creative Milestones

The timeline above (see Figure 1) provides a reference to the major milestones in facial and character animation and their application in the field of digital entertainment. As computer power advances and graphic capabilities progress, new possibilities have emerged for a wider creative expression through the production of more realistic models with believable expressions that convey human emotions. 
Author

\subsection{The Psychology behind the Face}

Psychology is an important part of facial animation, as it is the means by which we can study how humans understand and communicate emotion and therefore this may lead to a more creatively produced and realistic character.

Investigation into human perception of facial expressions has shown that there are visual signs that allow us to correctly interpret each expression. For example, anger has many defining points such as frown lines and a clenched jaw, which allow us to pinpoint the emotion that is being expressed.

The scientific study of facial expression of emotion can be dated back to French neurologist Guillaume Duchenne (1990). Duchenne investigated facial expression by using electricity to shock the facial muscles. He took photographs of his experiments to demonstrate how the facial muscles were used to create emotional facial expressions and mapped out 100 facial muscles (Duchenne, 1990). Certain facial expressions are associated with particular human emotions. Previous research has shown that people across cultures associate facial expressions with the same emotions. Charles Darwin (1872) was the first to make the suggestion that facial expressions are universal.

In the 1960s Paul Ekman conducted a series of experiments to test Darwin's claims. In 1969 he showed six photographs of different facial expressions to people in different continents and cultures worldwide, in which all attributed the same emotions to each facial expression (Ekman and Friesen, 1986). Ekman, Friesen, and Ellsworth (1982) reviewed several studies examining the perception of posed expressions in static photographs and found a high degree of consistency in the emotional categories that were assigned to such expressions. They concluded from these studies that there are six basic facial expressions: happiness, surprise, fear, sadness, anger and disgust.

Ekman and Friesen developed the facial action coding system (FACS) (Ekman and Friesen, 1978) to determine how contracting facial muscles change the appearance of the face. They developed the system by analysing photographs of faces in order to identify changes that occurred in muscular contractions, and differences between each expression. The FACS is a complex structure, which is measured in Action Units and not muscles. It was designed to deal with clearly visible expressions on the face, ignoring invisible changes (Ekman, 2007). FACS has become the most widely used method for measuring facial behaviours. In the film industry FACS has been used for realistic character animation. This is accomplished by recording the motion of a human subject, and implementing it onto a muscle-based character (Gralewski, 2004).

\subsection{Modelling Techniques and Facial Animation}

There are many methods by which facial expressions within 3D applications can be creatively produced. The most common methods are blend shapes, bones, motion capture, image and video based. These are presented briefly below.

\subsubsection{Blend Shapes}

Blend shape animation works by blending a source shape to a target shape. It is a key frame based method and works with entities such as vertices from a polygon 
mesh (Joshi et al, 2003). By gradually changing the blending percentage, the face changes from a neutral expression to the target expression, such as anger. Any number of targets can be applied to the base object, enabling the character to come to life to allow a more creative exploitation of different expressions. Typically blend shapes are controlled through sliders.

Animating using blend shape sliders is an easy and popular control method that encourages more creative exploration of a character's facial expression (Lewis and Anjyo, 2010). Using the principle of blend shapes Wang et al (2007) have developed a facial expression animation system by extending the FaceGem 3D authoring software that incorporates an off-the-shelf text-to-speech engine. Their system is mainly based on a text-to-speech synthesizer but it also includes a basic six facial expression control module.

\subsubsection{Image and Video-based}

Image based approaches generate 3D individual face model automatically using 2D full face images (Lee et al, 2005; Pighin et al, 2002). These approaches adjust the 3D facial model created and synthesize the facial model into various shapes. Video based approaches produce facial semi-automatic expression modelling and animation generation through moving images (Yin and Weiss, 2004; Xia et al, 2010; Manohar et al, 2010; Maejima et al, 2010; Malatesta et al, 2009).

Although these methods can be applied for facial expression modelling and animation, they are mainly suited for facial expression recognition. These solutions are available using custom tools and programming environments that make them inaccessible to amateur 3D users and creative individuals. Also these approaches although they show some promising results they require high technical expertise and do not facilitate further creative facial manipulation and generation of additional facial expressions from the ones captured.

\subsubsection{Facial Rigging with Bones}

Bones form another popular realistic expressions production process. A facial rig is employed to simulate muscles within a face. The animator typically creates bones which are connected by joints. These are positioned under the character's skin, similar to the human anatomy. When rigging the face, the bones are positioned in areas in which the animator wishes to create movement (Capell et al, 2005).

Facial animation using bones can be very problematic, especially in the light of the hundreds of different muscles that comprise the human face, which allow us to communicate expressions. Facial rigging allows the animator to use a vast amount of bones to create a very expressive face, but this in turn can lead to a more complicated control setup (Maraffi, 2003). Recent research developments (Orvalho and Susin, 2007; Baran and Popović, 2007; Zhang et al, 2004; Shi et al, 2008) claim a dramatic reduction in the facial rigging animation process. Still though such a technique requires a high level of technical knowledge and the great amount of time spent on developing and manipulating the control setup often detracts from the design and creative process. 
Author

\subsubsection{Facial Motion Capture}

Facial motion capture is a sophisticated technique, which speeds up the animation process by capturing facial expression in real time. The use of motion capture enables animators to edit and blend from multiple capture sessions, and mix and match them with key frame animation techniques, which allow control over the final output. Real time trackers, which are used for moving animation, consist of a very lightweight video camera that is trained onto the performers face. The camera records the two dimensional motion of a set of dots or markers that are attached to the face (Guenter et al, 2006). Current research in the field aims in combining blend shapes with motion capture data (Deng, et al, 2006; Li and Deng, 2008; Liu et al, 2008).

This technique can produce very realistic facial animation and allows creative expression of the facial animation. However it is inaccessible to the majority of creative individuals; it is relatively expensive and has high technical demands on capturing and post-processing (cleaning) of the captured facial expression animation.

\subsection{Related Software}

There are several commercial 3D design and animation software that enable users to creatively author and animate 3D faces. The most popular and widely used are Autodesk 3D Studio Max, Autodesk Maya, FaceGen Modeller, Motion Builder, 3DFXs, Faceporter and Magpie Pro. Examining each one of these in detail is out of the scope of this paper, however Table I provides a comparison summary of each.

Maya and 3DS Max form two of the most popular 3D animation software on the market, for both creative exploitation but also for high-end developers. Maya is mainly employed in the film industry but has become a strong contender in game development as well; whereas 3DS Max is mainly employed for the creation of characters in the game production domain.

\begin{tabular}{|l|l|l|l|l|l|}
\hline Description & FaceGen Modeller & Motion Builder & 3DFXs & Faceporter & Magpie Pro \\
\hline User Level & Novice & Intermediate & Novice & Advanced & Advanced \\
\hline Pros & $\begin{array}{l}\text { - prebuilt animation } \\
\text { expressions } \\
\text { - rapid creation of low } \\
\text { resolution character } \\
\text { animation }\end{array}$ & $\begin{array}{l}\text { - realistic facial } \\
\text { animation of } \\
\text { prebuilt and own } \\
\text { models }\end{array}$ & $\begin{array}{l}\text { - facial animation } \\
\text { creation based on } \\
\text { big library of } \\
\text { pre-built } \\
\text { expressions }\end{array}$ & $\begin{array}{l}\text { - exports } \\
\text { animation to } \\
\text { MPEG-4 }\end{array}$ & $\begin{array}{l}\text { - powerful lip- } \\
\text { sync animation } \\
\text { tool } \\
\text { - application } \\
\text { extensibility via } \\
\text { custom API }\end{array}$ \\
\hline Cons & $\begin{array}{l}\text { - Designed for } \\
\text { character animation } \\
\text { - User cannot create } \\
\text { own custom model } \\
\text { animation }\end{array}$ & $\begin{array}{l}\text { - requires } \\
\text { expensive motion } \\
\text { capture equipment }\end{array}$ & $\begin{array}{l}\text { - cannot export } \\
\text { models to other } \\
\text { 3D packages }\end{array}$ & $\begin{array}{l}\text { - cannot be } \\
\text { extended }\end{array}$ & $\begin{array}{l}\text { - specifically } \\
\text { designed for lip- } \\
\text { sync expressions }\end{array}$ \\
\hline
\end{tabular}

Table I. 3D Face Animation Software Comparison Summary

Both Maya and 3DS Max form large packages that can be cumbersome to learn. Maya's greatest feature, making it popular with professionals, is its fully customizable interface. It also has its own scripting language (MELScript) which 
is used to produce customized features (Alias, 2005). 3DS Max has also got a customized Graphical User Interface (GUI) and has its own scripting language (MaxScript), but it is not very popular or well documented in comparison to MELScript.

The progressive use of Maya in the film industry led to its popularity with creative users keen to produce similar 3D models and characters that they had seen on screen. A large number of 3D users employ Maya for its facial modelling tools. Maya's MEL scripting language allows the customization and creation of new interface components and plug-ins. This is why we have selected it to be employed as a use case for our proposed facial expression creation and animation technique/tool in this project.

\section{PROPOSED METHODOLOGY}

\subsection{System Overview}

Our proposed approach has been influenced by the interplay of psychology, anatomy and computing in the creation of facial expressions. More precisely, research behind the psychology of the face has revealed that there are six crossculturally accepted emotions that facial expressions contribute to. Certain facial expressions are associated with particular human emotions. This guided us in selecting the six key emotions as the basis for the creation of additional emotions through the cross-blending of these. Closer study of the work of Ekman and Friesen in the development of FACS has revealed the role of the anatomy of the face in the formulation of facial expression and hence the expression of emotions. It has helped us realise the key facial attributes in the formulation of facial expressions (e.g. eye brows, mouth, etc.) that would be manipulated by our proposed solution. Research into the various methods available for computer based animation and the relevant software has directed us towards the use of blend shapes and the bespoking of commercial authoring industry tools.

Following the aforementioned review of the different facial expression animation techniques, we have decided to adopt the blend shape technique. This is because this technique offers a graphical user interface that encourages more creative freedom when animating a diversity of facial expressions. In order to hide as much as possible of the underlying technical complexities of the facial modelling and animation process, we have proposed a tool that semi-automates the entire creation and animation process. This offers users a stronger expression of creativity, enabling them to focus more and have a greater control of the choreography of the facial expression production and animation. The proposed tool, named EasyAnime has been designed to tackle the technical and conceptual complexities that currently exist in professional 3D design and animation tools, such as Maya. For this reason it is comprised of two main components, namely the EasyDesigner and EasyStudio. The EasyDesigner forms a popup panel, with tools to aid the user in the creative course of modelling the head model to be animated. 
Author

Several of the tools, which are included in EasyDesigner, are found throughout Maya's interface. However by incorporating and grouping these tools into one window menu, it is anticipated that the creation process of the basic six facial expressions will be made easier and faster. EasyStudio forms the facial expression interface, which is employed by the user after the modelling of their basic facial expressions. The expressions are then imported into the EasyStudio where they can be further modified to suit the creative needs of the user and saved for animating and future use.

EasyAnime works within Maya as a plug-in, which is loaded on startup. EasyStudio and EasyDesigner are facilitated within EasyAnime. Although EasyAnime has been developed to run using Maya's MEL script environment, the methodology proposed here can be re-used and implemented to other popular professional 3D animation tools such as 3D Studio Max. EasyAnime is developed as a series of script files, which are installed by a simple drag-anddrop process and launched automatically. Figure 2 illustrates EasyAnime's main system structure. This depicts the major steps, from modelling the character and expressions, to launching EasyStudio. The creative processes have been mapped using a step-by-step approach, whereby each stage must be completed before the user can to move on to the next step. This facilitates artists and designers with a production workflow, which hides several of the tool's complexities and fosters creative project exploitation.

In the first step the user creates a neutral (no expression) 3D facial model of their intended character with the help of the EasyDesigner tool. In the second step, this is copied and pasted by the tool six times, one for each of the six primary facial expressions, so that the user can sculpt these six expressions onto the corresponding pasted 3D models. In the third step the blend shapes are semiautomatically created.

Once the face expression design stage has been completed, the user employs the face controller to create the face masks in a semi-automatic manner by selecting animatable points on the face. In the next step, the user employs EasyStudio to assign the attributes to the face mask by linking all the phoneme attributes to the mouth of the face mask. The user then selects and loads a character into the EasyStudio tool, which then automatically creates a number of expressions based on the six basic expressions created previously. These are then saved onto the user's computer or an external disk so that they can be re-used in future projects. Lastly the user employs the EasyStudio tool to animate these facial expressions. 


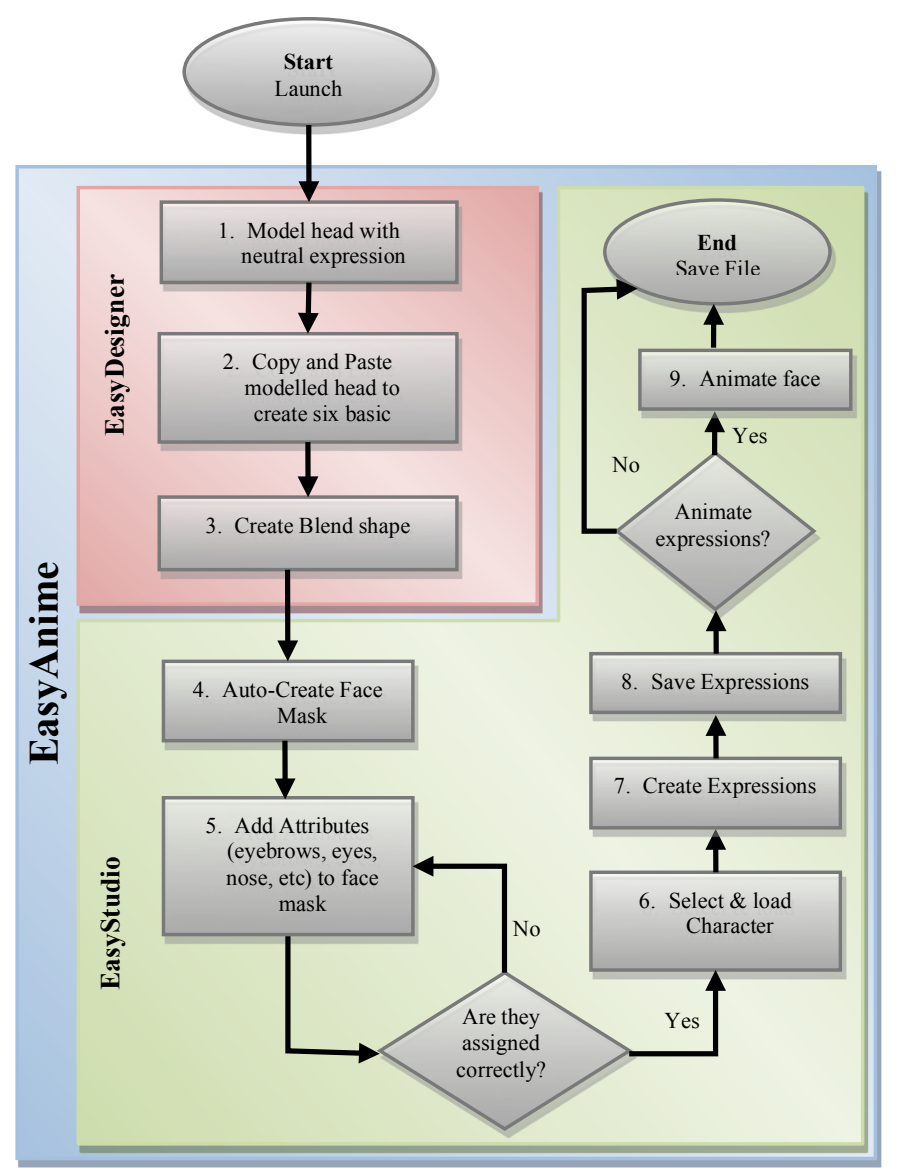

Fig. 2. EasyAnime's Main Structure Flow Chart showing the numbered steps

The workflow chart (see Fig. 3) shows the entire process from the start of the modelling process to storing the new expressions in the library and finally animating.

The modelling process begins by capturing a high-resolution image of the actor in a neutral pose. Next, the actor is sculpted into a 3D model. Each expression that is modelled can be stored in the expression database. The models use a standardized polygon mesh designed specifically to meet the demand of realistic facial expressions (thirty three thousand polygons smooth meshed). The database originally holds the six basic facial expressions and the phonemes that are modelled and then, by using the sliders in EasyStudio, new expressions can be created. The EasyStudio launcher searches for blend shapes when loading, where each of the blend shapes that exist for the model are depicted in a window in the expression library. The user animates the expressions, which are saved in the library for further manipulation and future use. 


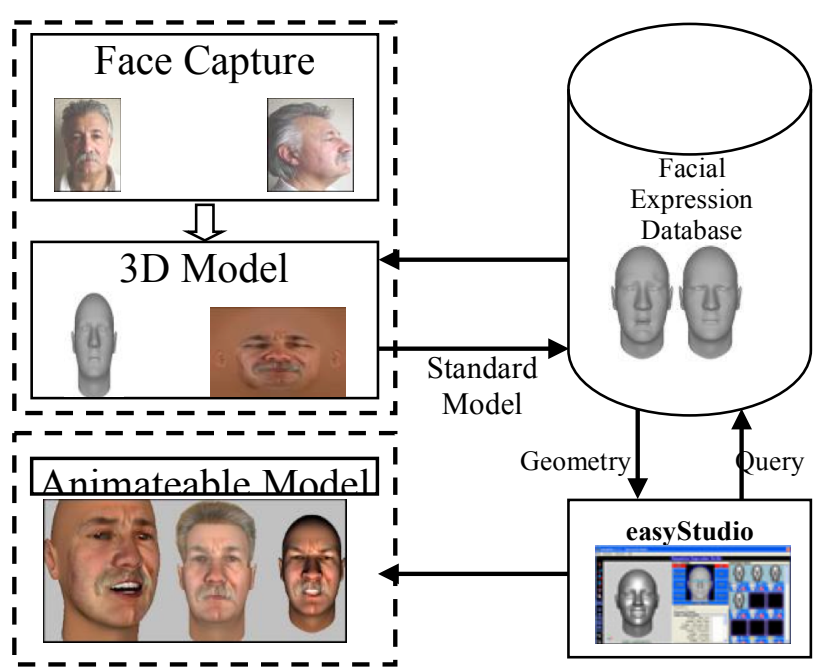

Fig. 3. Workflow overview

\subsection{Stage One - The Face Expression Design Stage}

In this stage (step 1 in Fig. 2) the user creates the face model that will be employed for the facial expression animation. It is important that the user creates a facial model with a neutral expression (i.e. no emotion to be portrayed at this stage). Once the neutral expression has been modelled, the head is duplicated for each of the six basic expressions (see Fig. 4).

For the purposes of testing the creative potential of the proposed tool two character models were created, namely a human character and a fictional character. In this paper we present the design of the human character. Photographs of an actual person were taken to act as realistic textures to make up the skin and teeth of the human character. This is a common technique for modelling from photographs or drawings.

In the next step (step 2 in Fig. 2) the face model created with the neutral expression is duplicated. Each of the duplicated heads is sculpted into one of the six basic expressions as shown in Figure 4. It is hypothesized that once the user has modelled the seven different faces or loaded pre-made ones (the six basic facial expressions and one neutral expression), (s)he will be able to create several other expressions by mixing various combinations. This will facilitate a large number of facial expressions to be created automatically; from which the designer can then select and use the ones that fit the most his/her creative aspirations and design project.

For the blend shape to work properly, the expressions must be duplicates from the original and most importantly have the same vertex count, because of the interpolation between vertices. Once each of the faces has been duplicated and the expression sculpted, the blend shapes can be created (step 3 in Fig. 2). By 
selecting one of the duplicate geometries and the original (neutral), the user is able to control the morph between the two shapes and thus modify the expression to suit their creative needs.

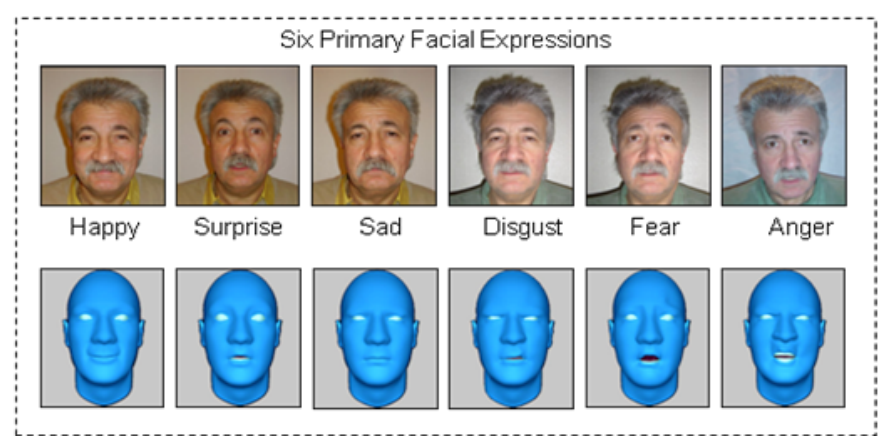

Fig. 4. Build diagram of test subject (Chris)

\subsection{Stage Two - The Face Expression Creation Stage}

In this stage (steps 4 and 5 in Fig. 2) the user creates the face mask, which facilitates the facial expression animation process. To enable this we developed the face controller, which is one of the main tools of the EasyStudio. It consists of a visual selection tool that enables the user to select animatable points on a face, and shows only the attributes connected to that particular facial feature. This is in contrast to the default Maya environment, which shows the entire list of attributes making the selection process cumbersome. The face controller speeds up the animation process and keeps the screen less cluttered.

The face mask is created by taking a photograph of the actor to be animated and automatically segmented into the important facial regions identified by Waters, 1987; Lee et al, 1995; Kähler et al, 2001, which correspond to the main muscles that play a critical role in human facial expression formation. The automatic face mask segmentation (step 4 in Fig. 2) hides the technical intricacies of such process allowing users to focus on the facial expression creation process. The face mask is a recognizable $2 \mathrm{D}$ face template commonly used to show face target points. This well-recognized face is ideal for the mask, as it has easily identifiable features and does not lean towards a gender. These features are depicted in Fig 4 and are: the eyebrows (A), eyes (B), nose (C), mouth (D) and chin (E). Once created, the face mask appears at the base of the user's character. When a facial expression is made, muscles are pulled and moved, which affect surrounding areas of the face. This information is translated onto the 3D model of the actor shown in Fig. 5 as coloured regions; this in turn renders it easier to identify the correct animatable parts for the human facial expressions. 
Author

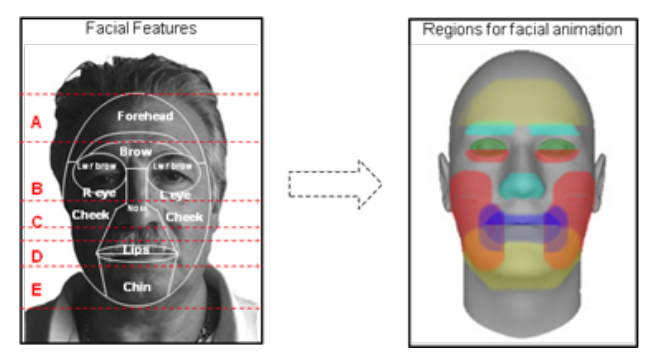

Fig. 5. Representations of Animatable Facial Regions

The next step (step 5 in Fig. 2) is to assign the attributes (eyes, eye brows, nose, mouth, cheeks, chin, and forehead) to the face mask.

The face controller enables the designer to specify the facial attributes in a straightforward 'click and select' manner without having to deal with the technical aspects of setting them up manually through the maze of buttons and options of Maya's original complex selection panel. Hence saving valuable time and effort that can be invested in more creative endeavours of facial expression production.

\subsection{Stage Three - The Face Expression Animation and Storage Stage}

In this stage (steps 6-9 in Fig. 2) the expressions are created and then saved onto a simple database (expression library) for future use. The expression library stores all new facial expressions created by the user. EasyStudio allows users to either work with the current character or load an already saved character.

Once the face mask and attributes have been applied to the character, the facial expression creation process can begin (step 6 in Fig. 2). Facial expressions are created (step 7 in Fig. 2) by blending the facial regions using the blending sliders available through the tool's user interface. A 'Create Pose' button copies the new expression from the viewer panel and saves a screenshot of the face into the expression library as a visual reference (step 8 in Fig. 2).

The library allows users to animate the expression either in a fully automatic animation mode using the "Auto Key" or in a semi-automatic manner using the 'Add Key Frame' command, which allows for further creative manipulation by the user (step 9 in Fig. 2). The new expressions created are seen as a screenshot in the user interface's working window. The user can click on any of them to save the selected expression.

\subsection{Implementation}

EasyAnime was implemented into the Maya program using MEL script. After EasyAnime is installed, it can be initialised from within Maya using the command line. Once installed a shelf menu and a file menu appear in the Maya UI environment both labelled "EasyAnime" (see Fig. 6).

The file menu is a text list of selection tools, which has the same controls as the ones found on the shelf menu, but with added shortcuts to the face mask and 
help menus. The shelf menus on Maya are icon based, with each image having its own functions, which are displayed in tabs across the top level of the shelf.
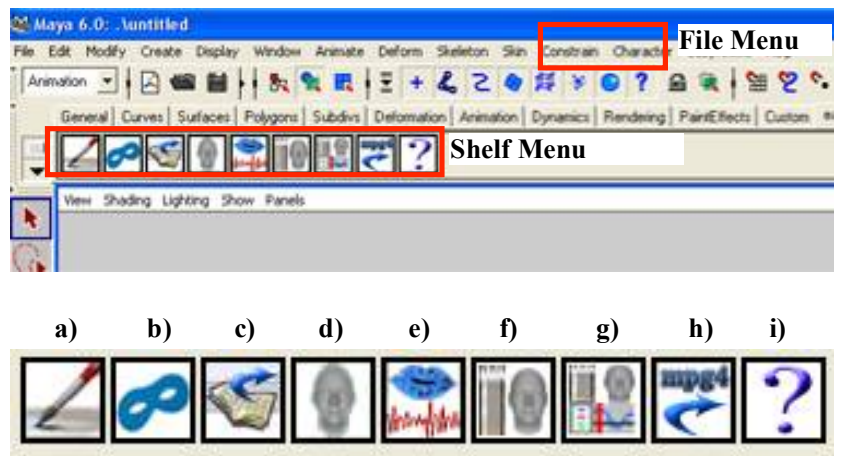

Fig. 6. Screenshot of the EasyAnime file, shelf menu and toolbar

The rows of icons shown in Fig. 6 appear under the EasyAnime shelf menu and are designed specifically for the new interface. The buttons are added for functionality and to launch procedures. The nine icons include: EasyDesigner (a), Create mask (b), Add Attributes (c), EasyStudio (d), Sound Manager (e), Layout 1 (f), Layout 2 (g) Mpeg4 Exporter (h) and Help (i). The Sound Manager and Mpeg Exporter buttons are disabled in this version, and will be developed in a later version. The EasyAnime menu shelf provides a visual toolset grouping in a hierarchical order (step-by-step production workflow starting from left to right) all the tools a creative individual would require for the completion of any facial expression and animation project.

Fig. 7 provides an overview of the system by illustrating its classes and relationships among them, in order to further understand the framework of the implementation of EasyAnime. The main class is the MEL Command Engine, which is directly associated with easyAnimeInstall. The EasyAnimeInstall has four main scripts associated with it, easyDesigner, easyStudio, easyStudioUI and easyAnimeHelp. Each of the inheritance class names work independently, except easyStudioUI and easyStudio, which are associated, as indicated by the dotted line. EasyAnime's application is written and read through the MEL Command Engine. All classes are associated with the easyAnimeInstall class. The classes' relationship with easyAnimeInstall is significant and must be initialized for any of the inheritance classes to work. This is accomplished by using Maya's command line and typing 'easy AnimeInstall'. 
Author

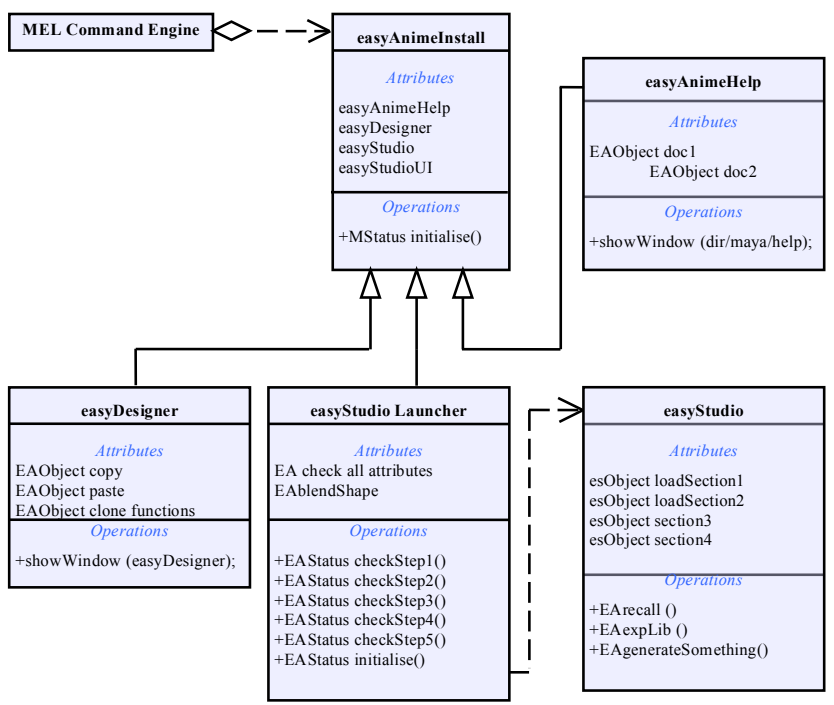

Fig. 7. Architecture of EasyAnime initialization in UML Diagram

EasyStudio includes five main operations/steps, which have been developed to automate the process once the application has been launched in Maya. These include checking the files, adding user assigned information, setting up the camera, loading the user interface and launching the user interface.

- In the first step the operation checks if a character has been selected, if it is in mesh format and has a joint attached and if blend shapes exists.

- In step two the operation collects the required information that has been assigned by the user; this includes the facial attribute groups and assigned blend shapes.

- Step three sets up the camera that is created with the face mask; this camera view is used as the main window in easyStudio. This includes setting the position and focal length of the camera by using pointConstraints and orientConstraints. The constraints are used to limit the object's position to the target object or to the average position of a number of targets.

- In step four the operation checks that the different editors exist in EasyStudio and that they can be launched correctly. If 'true' then a confirmation message is displayed in the text field, if 'false' then a substitute is being used.

- The final step launches the interface. Once all the steps are completed a message is displayed that "EasyStudio is being loaded"; then there is a three second pause to allow the launcher window to close, followed by the launch of EasyStudio.

It should be noted that the main script operations are declared by global procedures, which are followed by the function name (global proc 
$<$ function_name $>$ ). Global functions require more resources and are typically only used to call the main procedure. They have been used throughout the tool operations, as there are many individual procedures to be called within the interface, which is due to the way the system was developed.

Other important operations in the tool include the pressure sliders used for blending the shapes, the face controller and face mask, the expressions library and adding keyframes for the animation.

More precisely, a pressure slider is used to control the amount of pressure that is cloned onto the target shape. The script procedure is called showVertex and it is built using an intSliderGrp (integer slider group). The default behaviour of the slider and field is to have the same range of values specified with the $-\mathrm{mn}$ (minimum) and $-\mathrm{mx}$ (maximum) flags. Other flags that can be used in place of the previous flags are the -fmn (fieldMinimum) and -fmx (fieldMaximum) flags, which specify the larger range of field values. The values are set as percentages whereby $0 \%$ has no affect and $100 \%$ is a complete clone of the source shape.

The face mask script was developed by turning on the "Echo All Commands" option in the Script Editor, in order to find out which commands are executed as the mask is drawn. The mask is designed to appear at point $0,0,0$, which is the centre of the working environment, each of the curves are drawn separately and then grouped to form one mask. It is part of a much larger series of scripts, which create the mask automatically. Each series of the scripts has scale, move and xform commands. These three commands position the curves into place.

The expression library displays a collection of icon windows for each of the blend shapes that have been created. For each blend shape attributes are employed (using the addAttr function) to store all data in the scene. For every object that is added to the expression library a groupId is assigned and the number is incremented as more are added. The expression library also includes the blend shape controls. These use the attrFieldSliderGrp command. By default the command positions the controls in individual columns. The controls consist of a float field and a float slider, then the minimum and maximum values that are specified $(0,1)$.

Lastly the "add keyframe" command is employed for the animation of the character. The command, which is activated once the button is clicked, creates a key frame of the current blend shape attributes as a key shape on the timeline, which stores the current position of the slider.

\section{THE USER INTERFACE}

\subsection{EasyDesigner}

EasyDesigner was developed to provide extra functionality to the initial stages of modelling. When users of EasyAnime create their six basic expressions for their character, they firstly need to duplicate their model for each expression to be sculpted. Two tools have been implemented into EasyDesigner, in order to ease 
and speed up the modelling process. The first tool allows the user to copy the entire face onto another model. This is useful if the next expression to be modelled is similar to the previous. The second tool is used to clone specific areas of the source face to the target face, rather than copying the entire expression. EasyDesigner has incorporated the copy/paste and the clone tool together in the same window, as they are very useful tools to have on screen while modelling. Both tools have been modified in appearance and adapted to work with face models used in EasyStudio (see Fig. 8).

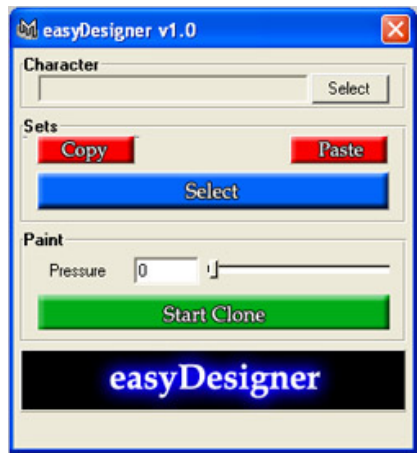

Fig. 8. The EasyDesigner Menu

One of these modifications is a simple selection tool, allowing the user to select their character's source shape before applying the copied vertices correctly to the duplicated model. This prevents the user having to use the right mouse button, shift key or the edit menu. The second major modification is the pressure slider, which has been added to the paint menu. The slider is used to control the amount of pressure used in the cloning process. For example, by increasing the slider to $100 \%$ allows an exact clone of the position of the vertices on the target face from the selected source face vertices. Taking the slider lower to $25 \%$ will allow the depth of the vertices to be positioned at $25 \%$ of the original depth. The slider eases considerably the design process as it enables the semi-automatic cloning of the facial expression, thus minimizing design repetition any manual manipulation form the user's perspective.

\subsection{EasyStudio}

EasyStudio's user interface is divided into four sections (Fig. 9). This section shows how each major section has been implemented into the EasyStudio user interface (UI). EasyStudio was developed in layers within one MEL script file, starting from the interface window that retains the menu icons and title bar architecture, followed by the individual sections. 


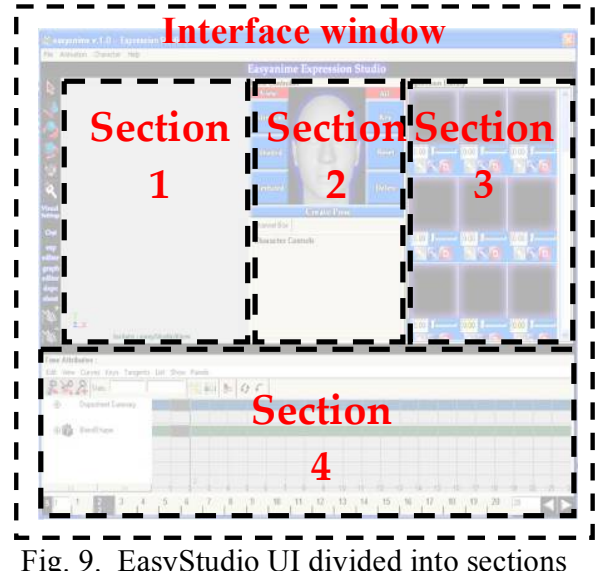

Fig. 9. EasyStudio UI divided into sections

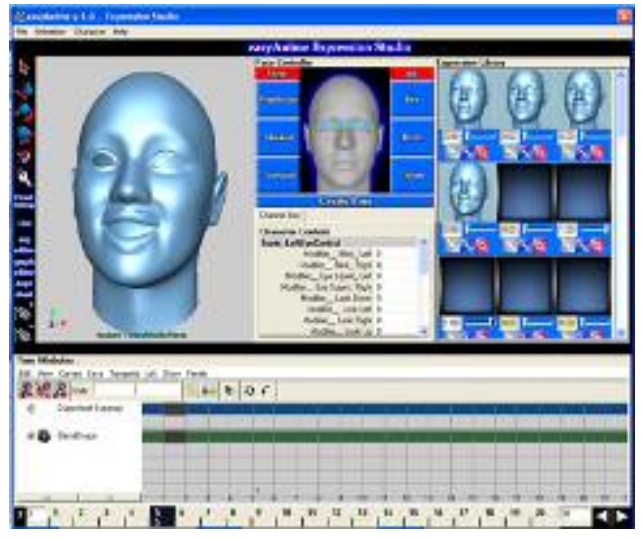

Fig. 10. EasyStudio UI in operation

\subsubsection{Section 1: The Viewer}

The Viewer, (see section one in Figure 9) is the working viewer window, it enables the user to see the manipulation of the facial expressions of their character. It is a clone of Maya's viewer window with the same tools to move, rotate and scale the face. The windows perspective view is taken from the camera created with the face mask, and any manipulation can also be seen in Maya's viewer. The window itself is labelled "easyStudioPane" and the camera view is labelled "easyStudioView". When the camera is created it has default attributes (focal length, zoom, scale), which can be changed and relabelled by the user at any time through Maya's tool panel.

\subsubsection{Section 2: The Face Controller}

The create mask menu contains one text field, which enables the user to name their character's mask, in order to easily save and locate it, in case more characters are to be added.

The attributes menu (see Fig. 11) consists of two lists. The left side contains the controls and the right side the list of attributes to be added. The tools are colour coded into sections and correspond to the order of the steps to be taken. The red buttons allow the user to add attributes and clear them. The second section contains the blue buttons, which allow the user to add the attributes to the specific section of the mask. They have been listed in the order they appear on the face. 
Author

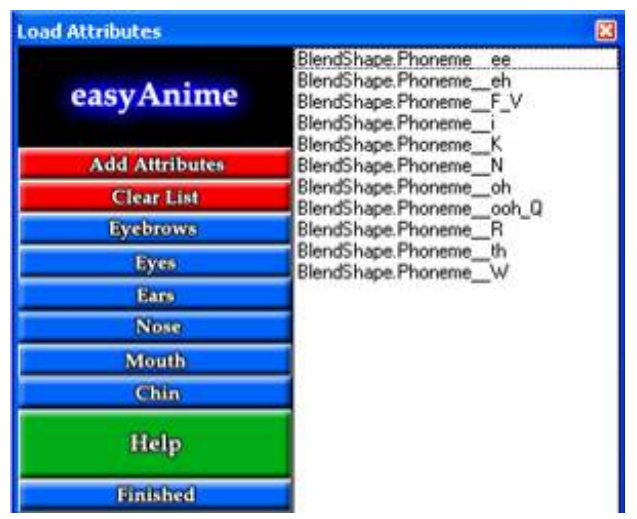

Fig. 11. The Attributes Menu operation

Fig. 10 shows the Face Controller panel located in EasyStudio (section 2). The face image is designed as a visual selector. For example, selecting the eye from the face image will show the attributes in the channel box that the user assigned in the previous attributes menu. When selecting any part of the face selector, a popup menu appears offering two choices: select and add. The select button highlights the area on the face with a box, the add button combines the selection with a previous selection in order to animate two or more features at the same time.

\subsubsection{Section 3 and 4: The Expressions Library}

The expression library stores all the expressions that exist for that particular model. The library is laid out as a visual gallery of the created expressions, which can easily be selected by the user. The library is saved onto the hard drive or storage device so that the expressions are available when the model is next loaded. This includes the data of the face (for example the position and pose not the actual model, as this is saved through Maya) and still images of the face to be used in the visual library in bitmap format. Additional 'Notes' can be stored within the file, for that particular expression or about the model itself and the design production in general.

\section{RESULTS AND DISCUSSION}

Testing was carried out to enable us to evaluate the creative potential of the proposed semi-automatic production of facial expressions technique in its current implementation in Maya. This was done in two stages. In the first stage of testing we evaluated the creative possibilities of producing several new expressions from the original six. This was achieved using different techniques of facial manipulation, looking at the quality as well as number of new facial 
expressions generated. In the second stage of testing we evaluated the proposed technique through user testing, by focusing on the tool's creative potential and usability. This provided us with invaluable feedback, which is presented below.

\subsection{Facial Expression Generation}

Testing under this section involves creating as many different expressions as possible from the six basic emotions, using alternative techniques in modelling and by using EasyStudio to blend the expressions. This facilitates the tool user with an increased creative output, as a plethora of new human expressions of the modelled character are created. For instance as we can see from Fig. 12 new expression that fulfil users' creative aspirations, such as being sad but surprised and disgust with anger being outputted.

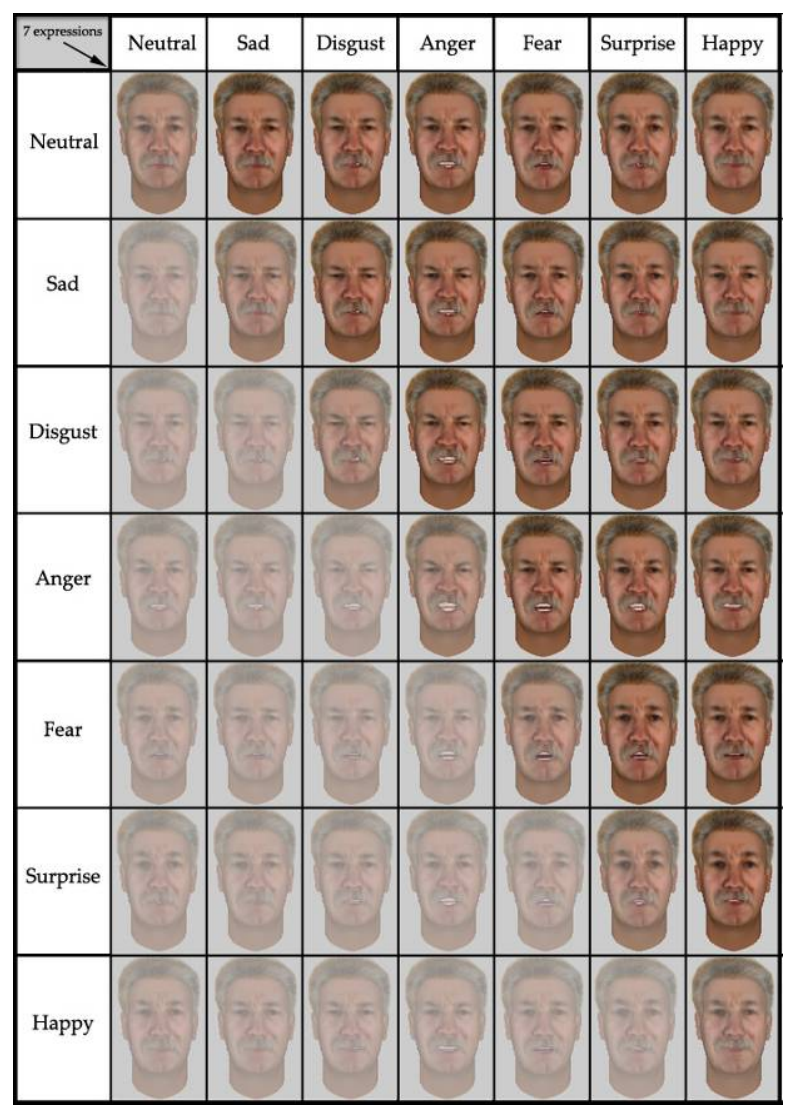

Fig. 12. Expression table of 21 new expressions created from the original six

In the default Maya Blend Shape editor tool, mixing a $50 \%$ blend of an expression with $50 \%$ of the corresponding expression yields a new expression. This is the most common way to blend expressions, which can be tuned using the sliders to increase or decrease the mix of the expression. Figure 13 illustrates the seven basic expressions that were modelled originally, running diagonally from 
top left to bottom right. The faces that can be seen clearly are the new expressions created from the original seven. The faded faces are the duplicated expressions that are created when blending in a grid table. After cross blending the expressions with each other, a further 21 expressions are created from the original ones, as seen in Figure 12.

Using the EasyDesigner tool, a different technique is applied to create even more expressions from the original seven. This is achieved by duplicating each expression six times, then by using the clone tool the lower level of each expression is copied to the bottom of a different expression. This is repeated until all of the different combinations are altered. Another technique to achieve the same result, especially if the model has a different vertex count to the source shape, is to divide each expression into two physical halves, which are then correspondingly labelled "upper" and "lower" part.

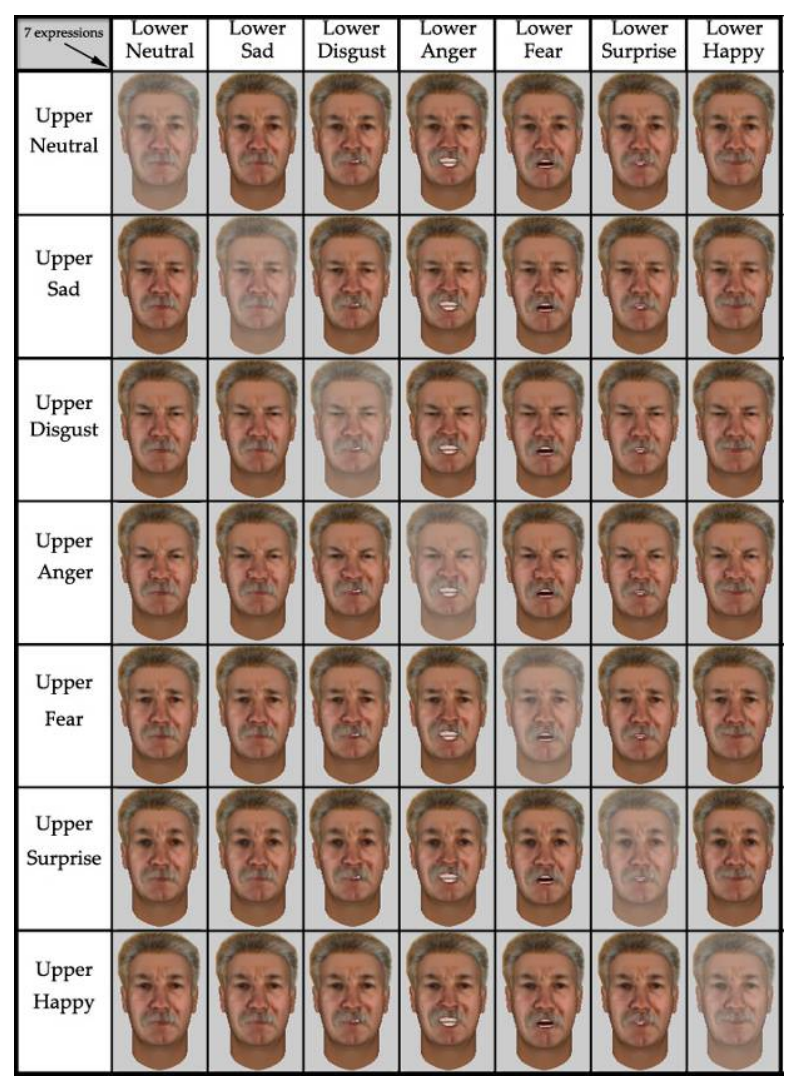

Fig. 13. Expression table of 42 new expressions using the upper and lower parts method created from the original six regions

The lower part of each expression is then matched to every upper at least once and the two halves are welded together to form the new expression. Each face should correspond to the blend of the two expressions in the same grid formation used in Fig. 12. This new technique can be seen in Fig. 13. The faded faces are the original poses and therefore are not counted as new expressions. 
By dividing the face in two autonomous areas of upper and lower parts, 42 new expressions are created, as seen in Figure 13. These faces could again undergo the same procedure, by blending them in EasyStudio and creating a mix between the two once more. The face can be further divided into three segments of main facial features: brow and eyes, cheeks, ears and nose and finally chin and mouth. Mixing between combinations of these parts would generate a further 336 facial expressions.

This approach and tool output offers a greater expression of creativity as it enables users with little or no technical knowledge to quickly produce a vast range of realistic facial expressions that can fulfil their creative curiosity. Especially by being able to segment the upper and lower half of the face, interesting combinations of facial expressions of more rich emotional meaning are created; such as upper fear with lower sadness or lower happy with upper surprise, which allow a finer control of the choreography of the face and can be used in any artistic project.

Although there is a limit to blending between so few expressions, the initial goal is to allow a creative and amateur user to focus simply on building a few expressions in order to animate their character to a high standard in a short amount of time and in a creative manner. This is achieved by modelling the face and then by sculpting the six basic human emotions. The user is then able to create a sufficient amount of expressions using different blending techniques accelerated by the EasyStudio tools. As one can see in fig. 14 and 15 the pool of new expressions created from the basic six in a semi-automatic manner provides designers, artists and creative individuals in general with the freedom and flexibility to pick the facial expression suiting the most their production and encourage creativity.

This enables users to create an animation of a very high standard, which can be saved within the expression library and altered for future projects too. This alternative method would prevent consuming valuable time on modelling expressions, since they can now be sculpted from pre-existing faces. In addition, the possibility of creating facial expression with various degrees of discrepancy is a critical part for any facial expression and animation project, as viewers are able to distinguish and ascribe different meaning to nuances in the human facial expressions.

\subsection{Phoneme Generation}

To support further user's creative needs we also investigated the generation of phonemes. To provide the appearance that a character can talk, phonemes need to be sculpted for each of the sounds of the English Language. Animating or blending with phonemes works in the same way as working with emotions. Using the expression library within EasyStudio, the user is able to mix phonemes with expressions to accurately bring the character to life.

In most $3 \mathrm{D}$ projects this is achieved by either developing the phoneme face pose with an already expressive upper segment or by creating separated controls for the attributes on the face. However these approaches are on one hand very 
difficult to achieve for non-technical savvy users and on the other hand are extremely time-consuming, as they demand the setup of a rig, which controls each attribute on the face. On top of this, these approaches are not always possible to incorporate that early in the face creation process, due to the difficulty in knowing the exact elements that will be involved in the final animation project, such as the facial poses, dialogue and so on. These important creative decisions are often taken and decided at a later stage. Therefore a method that supports the creative process a better should be employed.

An alternative approach is to use the blending facilities, which the EasyStudio tools employ as well. Testing of this approach revealed that the new expression sometimes lost the detail of the original sculpted phoneme. This is due to the vertices being moved too much, resulting in a loss of detail in shapes of the mouth.

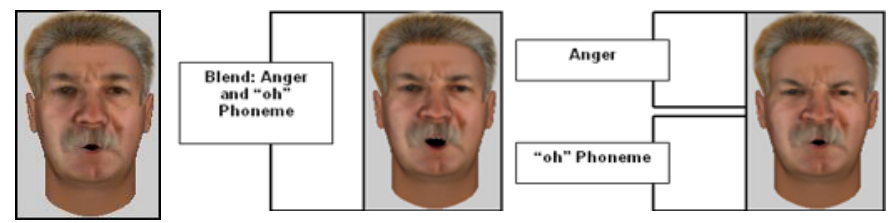

Fig. 14a. "oh" phoneme Fig. 14b. Anger \& "oh" phoneme blend Fig. 14c. Split expression \& weld vertices

Figure 14a shows the original sculpted phoneme for the "oh" sound. Blending the shape within EasyStudio with an "Anger" expression, for example, generates a new expression (Fig. 14b). It is clearly visible that the shape of the mouth changes with the blend, and with other phonemes, the shape can be lost entirely. However, by dividing the face into three segments (brow and eyes; cheeks, ears and nose; chin and mouth), the phoneme can be kept on the lower level and the expression in the eyes and brow can be kept in the upper level, producing a much more realistic and expressive facial expression without losing any of the detail (see Fig. 14c). However, this is dependent on the expression and the strength of emotion the user wants the character to illustrate. This technique provides a much wider scope of emotions and speech that worth investigating further and that can be employed in even more creative productions such as games, movies, digital performance where the actor interacts with a virtual avatar and many more.

The phoneme generation example described and tested above was applied to a single phoneme. In the English language however there are 38 phonemes. The only types of sounds that relate to animation are the sounds made by the lips. Animation speech is ruled by a system known as 'visual phonemes', or visimes. The use of Osipa's method reduces the 38 sounds for speech to just 7 to sufficiently cover enough phonemes to give the appearance that the character can talk (Osipa, 2007). These can be modelled using photographs or a mirror as reference for each of the phonemes to be sculpted, resulting in even fewer poses needed to be sculpted. 


\subsection{User Testing and Feedback}

To test and evaluate the EasyAnime concept, the proposed tool was sent to amateur/novice but enthusiastic users with basic modelling skills. The aim was to carry out a qualitative study to investigate whether the tool supports creativity and how usable it is. These were measured based on the time it took to complete the assigned tasks, the questionnaire feedback, the user feedback and observation notes.

In addition to this and in order to further test the EasyAnime concept and compare its usefulness with other 3D commercial animation tools, we examined facial expression creation and animation in Autodesk 3DS Max too. More precisely, we provided participants with a model with four of the six basic expressions already sculpted in 3DS Max file format and asked participants to animate a face in 3DS Max too. It should be noted here that 3DS Max does not have a dedicated facial animation tool, such as Maya. The closest equivalent is the Morpher modifier with custom built face controls connected to morphs through parameter wiring. The reason for selecting 3DS Max was due to the high popularity of the software by novice users and the different workflow with regards to facial animation, which facilitates a wider comparison option.

25 participants were recruited that fitted the experiment criteria, of which half were carried out under observation. All participants were novice users from a creative background and were familiar with Autodesk Maya and/or 3DS Max. Participants were divided into two groups based on their previous experience with either Maya (13 participants) or 3DS Max (12 participants).

Novice users here are defined as those who along with a creative aspiration have basic modelling, texturing and animation skills, thus they can model, texture and animate a low-poly 3D model using spline/poly modelling and animate it using each 3D authoring environment's auto-key and semi-automated animation tools.

The tests involved three tasks, which users had to follow from the given instructions under timed conditions, which were recorded at the end of each task, as well as a questionnaire to be filled at the end of the study.

To prevent the study from being tedious, the participants were given a model with four of the six basic expressions already sculpted, which allowed them to start from the point of creating the remaining two. The participants were sent a zipped file containing the MEL files, along with full instructions on how to install and initialize EasyAnime. The three tasks given to participants were selected to reflect the most creative aspects of the tool and are summarized below:

- Task one: Sculpt the remaining two expressions and add them as blend shapes to the original.

- Task two: Create a further 10 unique facial expressions and save them ready for animating

- Task three: Animate a 25 second short video clip of the face morphing into new expressions created, using the animation tools. 
At the end of the experiment users were asked to repeat the test on the remaining interface (Maya or 3DS Max depending on the group they belonged to). Each participant recorded their times, which were retrieved along with the 10 new facial expressions and the 25 second clip.

\subsection{Results and Discussion}

Participant observation was very useful in helping us gain insights in the actual use of the tool. We noted that users adopted a number of different approaches to carry out the same tasks. The more experienced participants of the group preferred to install and figure the new interface out for themselves rather than follow the instructions. The less experienced ones followed the instructions more closely, going through the interface in a procedural manner.

Fig. 15, Fig. 16, Fig. 17 and Fig. 18 show the results obtained by participants carrying out the specific tasks on Maya's Blend Shape Editor, 3DS Max and EasyAnime. Looking at all four figures one can see that overall participants required more time to complete all three tasks using the 3DS Max's Morpher Modifier and Maya's default Blend Shift editor tool when compared to EasyAnime. Also it is clear that participants tool longer to complete tasks on 3DS Max when compared to Maya. Looking at task 1 and task 2 (see Fig 15-18) a clear distinction can be seen between all three animation environments.

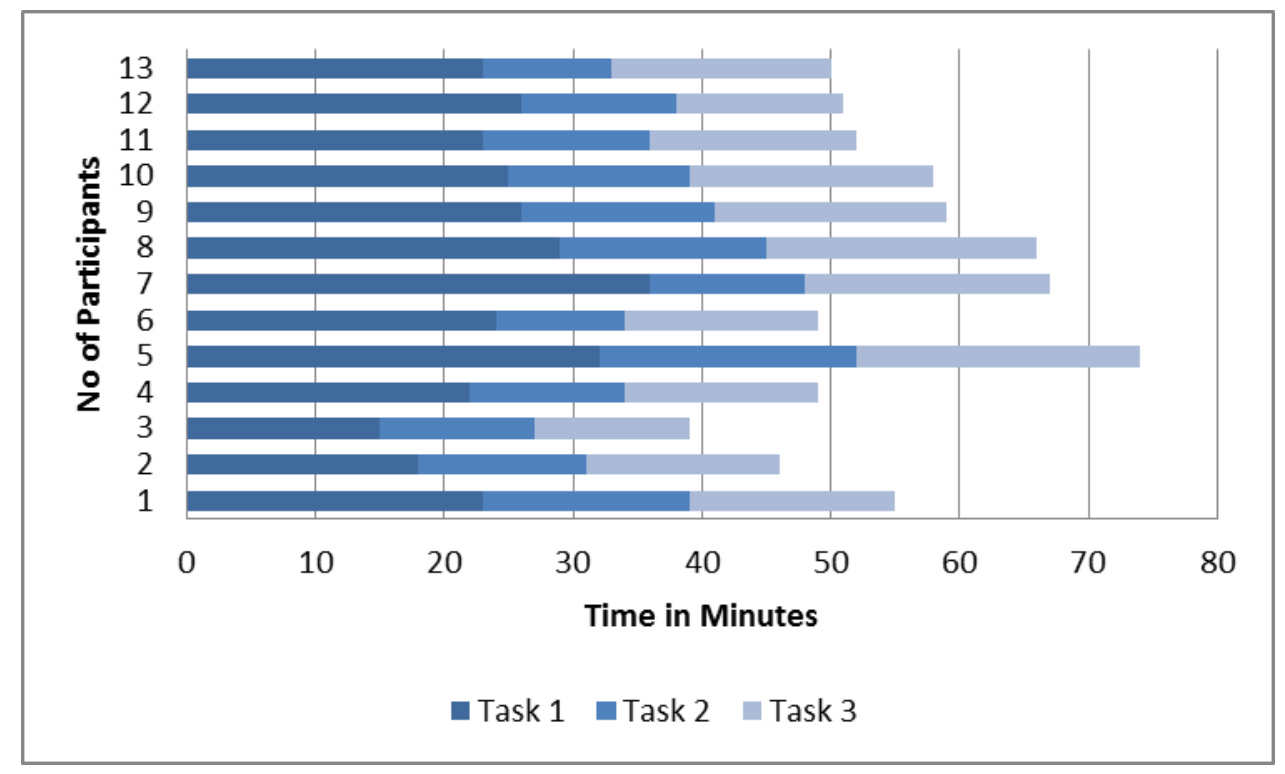

Fig. 15. Outcomes of timed test carried out on Maya Blend Shape Editor. 


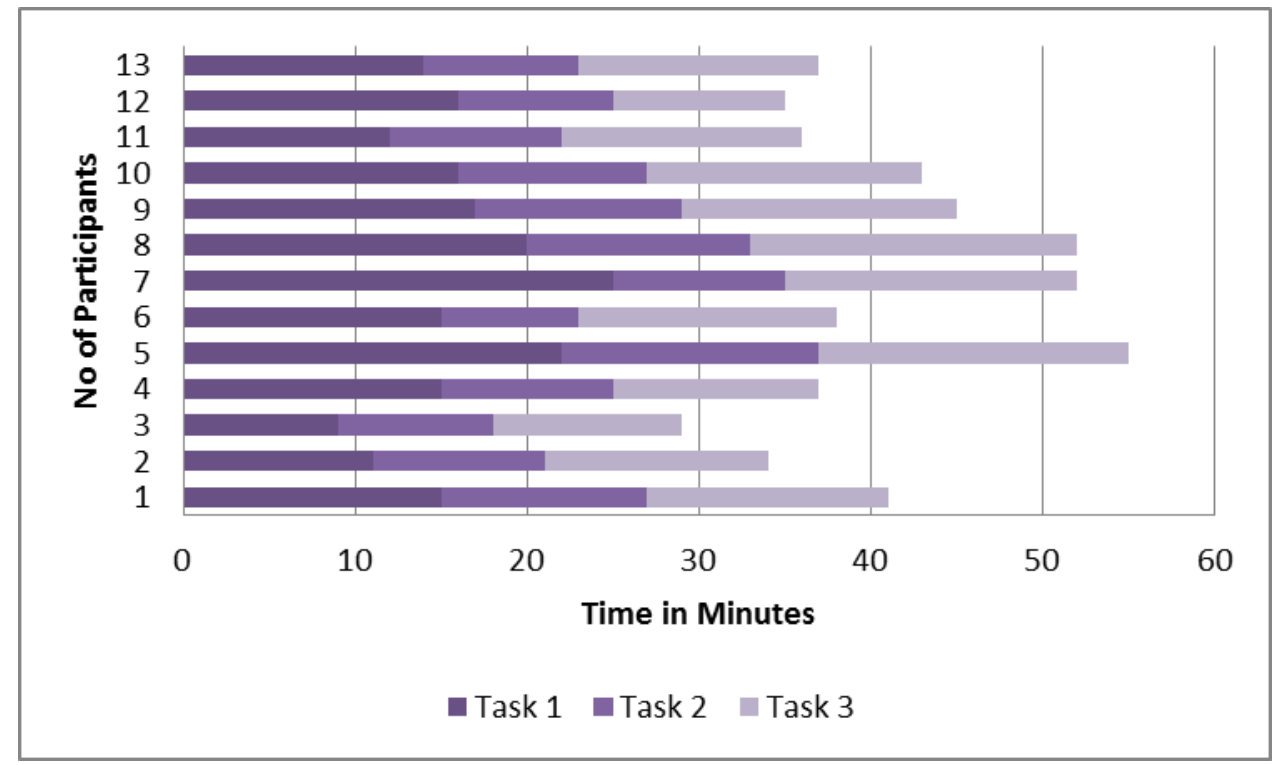

Fig 16. Outcomes of timed test carried out on EasyAnime's Interface - Maya Users.

When it comes to sculpting of facial expression users employing EasyAnime took 8 minutes less compared to performing the same task using Maya's default tool (Blend shape editor) and 3DS Max's equivalent tool.

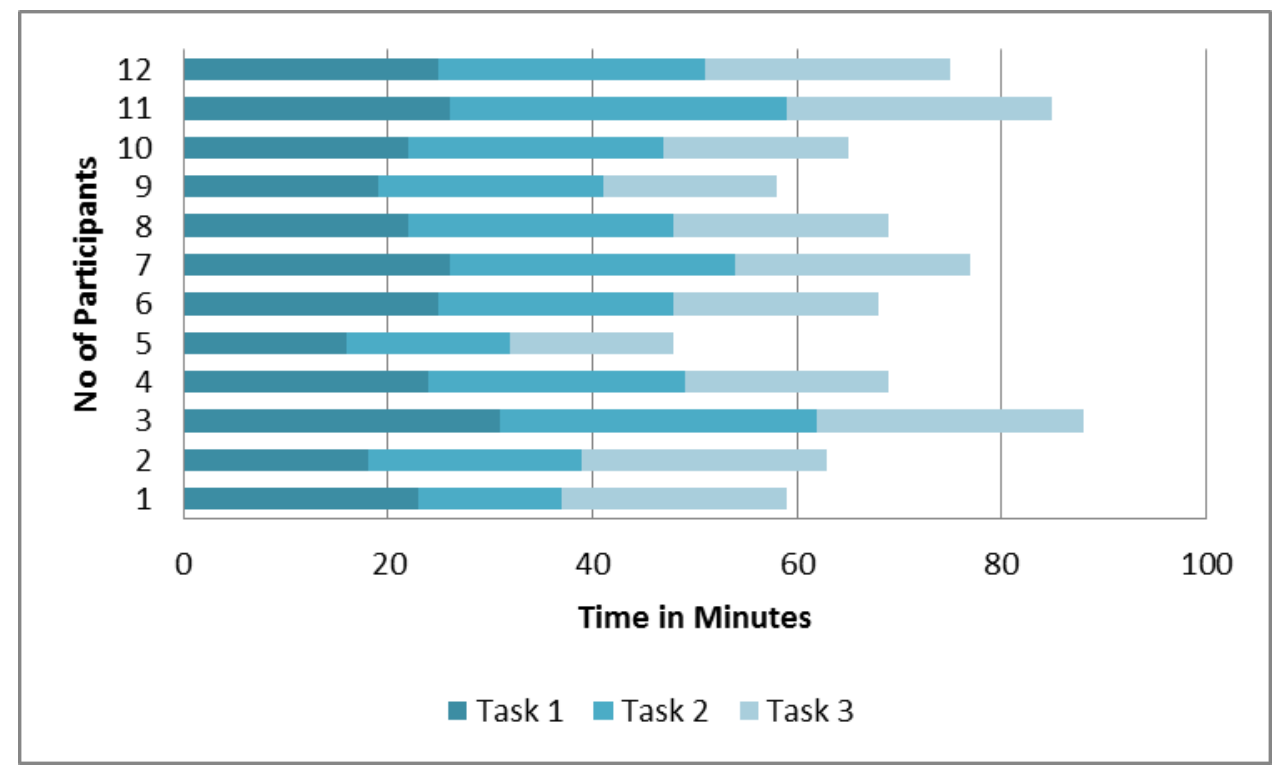

Fig. 17. Outcomes of timed test carried out on 3DS Max (Morpher Modifier). 


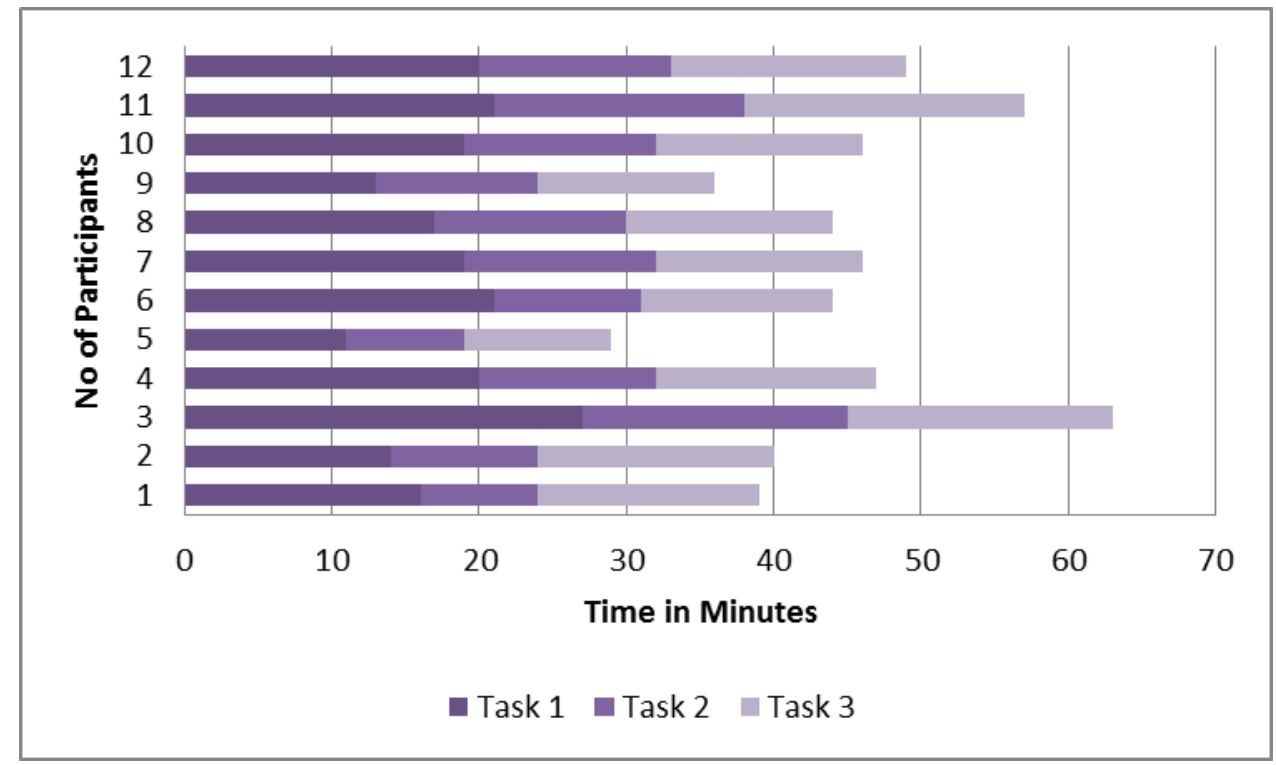

Fig 18. Outcomes of timed test carried out on EasyAnime's Interface - 3DS Max Users.

This time saving is due to all participants under observation making use of the new tool, which decreased the modelling time of the two remaining expressions. The Expression Library, with the built-in blend shape slider, was admitted to be an easier morphing tool compared to the Maya default one. This was said to increase creativity by cutting down participant effort and time when creating and saving the new expressions. The results were promising for Task 2, as when compared to the original Maya Blend shape editor and 3DS Max. In particular EasyAnime users saved an average of three minutes when compared to Maya's blend shape editor and nearly five times that (14 minutes) in contrast to using 3DS Max. The face controller was found as a very useful feature; however participants spent most of their time under this task in setting the attributes to the face in preparation for animation. Therefore one conclusion here is that automating the attribute assignment process would have supported further productivity and creative exploration of facial expression creation.

In Task 3 (facial expression animation), completion times were similar to the Maya's default interface. This is due to the animation tools being very similar. The outcomes are very different when one looks at the time taken participants to complete the same task in 3DS Max, where it took six minutes. Looking at the times taken participants to complete the last two tasks in 3DS Max, one can realize that developing an equivalent EasyAnime tool in 3DS Max (using 3DS Max native scripting language - MaxScript), which semi-automates this process in 3DS Max would have reduced times spent on these tasks significantly.

Table II provides an overview of the average times taken to complete all three tasks. The original (Maya's Blend Shape Editor) interface took, on average, 55 minutes to complete all three tasks, whereas it took 41 minutes on average, to 
complete the same tasks in EasyAnime and 68 minutes respectively on 3DS Max morpher modifier.

On average EasyAnime users spent 14 minutes less in creating and animating facial expressions. This results to a production time reduction of approximately $25 \%$. This percentage increases significantly when looking at the overall time difference between 3DS Max and Maya; where a 40\% production time reduction is evident.

\begin{tabular}{llllllllllll}
\hline Tasks & $\begin{array}{l}\text { Maya } \\
\text { Average }\end{array}$ & $\begin{array}{l}\text { Maya } \\
\text { Std. } \\
\text { Dev }\end{array}$ & $\begin{array}{l}\text { 3DS } \\
\text { Average }\end{array}$ & $\begin{array}{l}\text { 3DS } \\
\text { Std. } \\
\text { Dev }\end{array}$ & $\begin{array}{l}\text { EasyAnime } \\
\text { Maya users } \\
\text { Average }\end{array}$ & $\begin{array}{l}\text { EasyAnime } \\
\text { Maya users } \\
\text { Std. Dev }\end{array}$ & $\begin{array}{l}\text { EasyAnime } \\
\text { 3DS users } \\
\text { Average }\end{array}$ & $\begin{array}{l}\text { EasyAnime } \\
\text { 3DS users } \\
\text { Std. Dev }\end{array}$ & $\begin{array}{l}\text { EasyAnime } \\
\text { Average }\end{array}$ & $\begin{array}{l}\text { EasyAnime } \\
\text { Std. Dev }\end{array}$ \\
\hline $\begin{array}{l}\text { Task } \\
1\end{array}$ & 24.92 & 5.68 & 23.08 & 4.07 & 16.08 & 4.54 & 18.17 & 4.3 & 17.13 & 4.4 \\
$\begin{array}{l}\text { Task } \\
2\end{array}$ & 13.75 & 2.66 & 24.17 & 5.5 & 10.75 & 1.95 & 12.17 & 3.09 & 11.46 & 2.62 \\
$\begin{array}{l}\text { Task } \\
3\end{array}$ & 16.75 & 3.07 & 21.42 & 3.34 & 14.58 & 2.77 & 14.67 & 2.46 & 14.63 & 2.51 \\
Total & 55.42 & & 68.67 & & 41.33 & & 44.83 & & 43.08 & \\
\hline
\end{tabular}

Table II. Study Average Completion Times (In Minutes)

It should be also noted that the standard deviation is fairly small indicating that users took task completion times were fairly consistent across. With a few refinements based on feedback received from participants production times and focus on the more creative aspects of the production process may increase even further. The majority of participants emphasized the proposed tool's potential for the creative community. They indicated that on one hand the semi-automatic step by step workflow allowed them to focus more on the facial expression design and animation and on the other hand the production time reduction can provide them with more time for creative exploration.

The questionnaires completed at the end of the study assisted us in gaining an understanding of the proposed tool structure and creative potential compared to the original. Participants were asked to rate all three tools using a five point scale questionnaire design. Fig. 19 illustrates the questionnaire main results. The results show a clear distinction is some areas whereas in other ones EasyAnime falls short of the Maya Blend shape editor or 3DS Max. Looking across the different questions/fields we can see that participants preferred the new proposed tools over Maya's default ones and 3DS Max's morpher modifier. The animation tools did not differ from the original and participants found it equally as easy to animate. 


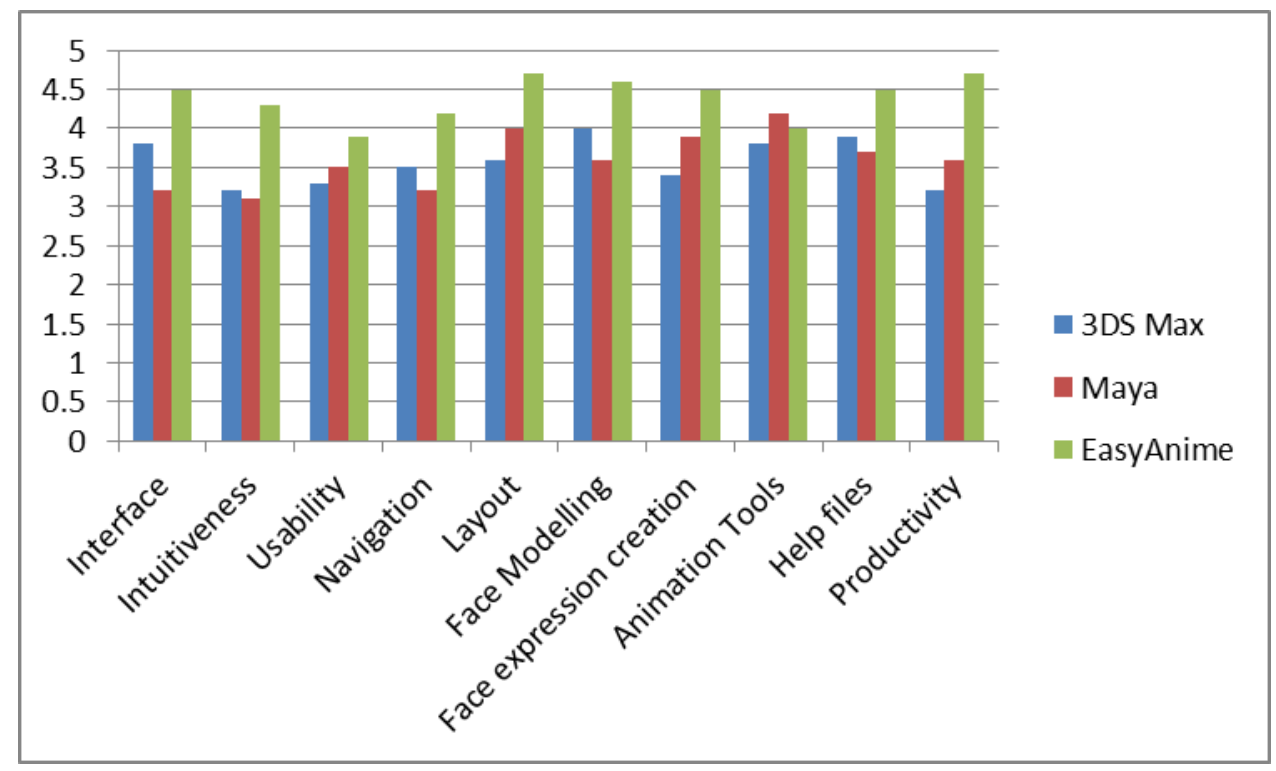

Fig. 19. Study Questionnaire Results

The help files that were included within the interface were found to be extremely useful to the users when they encountered difficulties. Most of these problems presented when using the attribute menu, which was anticipated and therefore an extra help menu was built within the window. The participants found the EasyStudio's help pages more useful than the original, as Maya's inbuilt help files are incomplete and very generic in this area, although alternative help can be found online. Last but not least EasyAnime outscored Maya and 3DS Max in terms of productivity, intuitiveness and usability.

\section{CONCLUSION}

This paper has presented a technique and an associated tool for enhancing and facilitating creative users with the means of producing of realistic facial expressions and animation.

In today's digital media renaissance there is the possibility of creating facial animation using existing commercial tools. However creating realistic and believable facial expressions, which can be explored in different creative environments and applications, is still beyond the reach of individuals who are willing but do not have the required technical expertise. The difficulty in the creation of realistic facial animation lies in the technical challenges associated with the creation of realistic facial expressions, which is usually only achieved by expert users with the relevant experience. As (Hugill and Yang, 2013) indicate creative users are typically forced to adapt their own creative practices when working with software. The proposed technique presents a step towards the adaptation of software to suit the creative practices of individuals. 
Drawing from multi-disciplinary research (anatomy, psychology, computer science) we employed a semi-automatic production process of facial expressions and animation that hides the technical challenges and constraints. As a use case we have implemented the technique to create the EasyAnime tool, which sits within Maya. It aids the user in the creative process of modelling the six facial expressions, which correspond to the six basic human expressions. From these initial six expressions several more are created by using different shape blending techniques, allowing the user to have more creative control on different parts of the face. This facilitates the generation of both common as well as new realistic facial expressions with distinct emotions being portrayed, such as fear with surprise or having the upper part of the face show surprise and the lower part show disgust. This emotion is a typical example of the creative flexibility and experimentation that the output of the EasyAnime tool provides.

Our facial expression generation and visime animation results and user testing indicated that the proposed technique and tools facilitate creative but novice users in producing a diversity of both common but also interesting (when combined different emotions for the upper and lower parts of the face) realistic facial expressions.

The user testing indicated that the proposed technique allows users to successfully model further facial expressions prepared for animating, faster than Maya's Blend Shape Editor by introducing an average of $25 \%$ production time reduction as well as 3D Studio Max's equivalent tools by introducing an average of $40 \%$ production time reduction. It is therefore expected that implementing the proposed technique, in other 3D authoring tools, such as 3DS Max's could potentially offer similar benefits.

Future work will aim at simplifying the facial attributes menu further to fully automate the process for faster development and also develop a dialogue tool to sync character's lips to speech. The later will be the main focus of the next version of EasyAnime. In addition more work will be conducted in adopting the proposed technique and developing EasyAnime equivalent tools in 3DS Max and Blender.

\section{REFERENCES}

Baran, I., and Popović, J. 2007. Automatic rigging and animation of 3D characters. In Proceedings of ACM SIGGRAPH 2007 Papers. San Diego, August 2007. 72

Borshukov, G., and J. P. Lewis. 2003. Realistic human face rendering for "The Matrix Reloaded". In Proceedings of SIGGRAPH conference Sketches \& applications. San Diego, July 2003. 1-1.

Capell, S., Burkhart, M., Curless, B., Duchamp, T., and Popović, Z. 2005. Physically based rigging for deformable characters. In Proceedings of the 2005 ACM Siggraph/Eurographics Symposium on Computer Animation Los Angeles, July 2005. 301-310.

Darwin, C. 1872. The Expression of the Emotions in Man and Animals. Murray. London.

Deng, Z., Chiang, P., Fox, P., and Neumann, U. 2006. Animating blendshape faces by crossmapping motion capture data. In Proceedings of the 2006 Symposium on interactive 3D Graphics and Games. California, March 2006. 43-48. 


\section{Author}

Duchenne, G.B.A. 1990. The Mechanism of Human Facial Expression. Cambridge University Press.London.

Ekman, P. 2007. Emotions Revealed: Recognizing Faces and Feelings to Improve Communication and Emotional Life. Macmillan. New York.

Ekman, P., and Friesen, W. V. 1978. Facial action coding system: A technique for the measurement of facial movement. Consulting Psychologists Press. Palo Alto, California.

Ekman, P., and Friesen, W. V. 1986. A new pan cultural facial expression of emotion. Journal of Motivation and Emotion, 10, 2. 159-168.

Ekman, P., Friesen, W. V., and Ellsworth, P. 1982. Emotion in the human face. Ekman, P. (Ed.), 2nd Edition. Cambridge University Press. New York.

Gralewski, L., Campbell, N., Thomas, B., Dalton, C., Gibson, D., and Bristol, U. O. 2004. Statistical synthesis of facial expressions for the portrayal of emotion. In Proceedings of the 2nd international Conference on Computer Graphics and interactive Techniques in Australasia and South East Asia. Singapore, June 2004. S. N. Spencer, Ed. GRAPHITE '04. ACM, New York. 190198.

Guenter, B., Grimm, C., Wood, D., Malvar, H., and Pighin, F. 2006. Making faces. In Proceedings of ACM SIGGRAPH 2006 Courses. Boston, July/August 03, 2006. 18.

Huang. D. Lin. W, and Yan. W. 2001. Modeling of Facial Expressions Using NURBS Curves. In Proceedings of the 6th International Computer Science Conference on Active Media Technology (AMT '01), Jiming Liu, Pong Chi Yuen, Chun Hung Li, Joseph Kee-Yin Ng, and Toru Ishida (Eds.). Springer-Verlag, London, UK, UK, 216-223.

Hugill, A. and Yang, H. (2013) 'The creative turn: new challenges for computing', Int. J. CreativeComputing, 1, 1, 4-19.

Joshi, P., Tien, W. C., Desbrun, M., and Pighin, F. 2003. Learning controls for blend shape based realistic facial animation. In Proceedings of the 2003 ACM Siggraph/Eurographics Symposium on Computer Animation San Diego, July 2003. 187-192.

Kähler, K., Haber, J., and Seidel, H. 2001. Geometry-based muscle modeling for facial animation. In No description on Graphics interface 2001 (GRIN'01). Canadian Information Processing Society, Toronto, Ont., Canada, Canada, 37-46.

Lee. H., Kim. E. S, Hur. G. T, and Choi. H. Y. 2005. Generation of 3D Facial Expressions Using 2D Facial Image. In Proceedings of the Fourth Annual ACIS International Conference on Computer and Information Science (ICIS '05). IEEE Computer Society, Washington, DC, USA, 228-232.

Lee, Y., Terzopoulos, D., and Walters, K. 1995. Realistic modeling for facial animation. In Proceedings of the 22nd Annual Conference on Computer Graphics and interactive Techniques. S. G. Mair and R. Cook, Eds. SIGGRAPH '95. Los Angeles, August 1995. 55-62.

Lewis. J. and Anjyo. K. 2010. Direct Manipulation Blendshapes. IEEE Comput. Graph. Appl. 30, 4, 42-50.

Li, Q. and Deng, Z. 2008. Orthogonal-Blendshape-Based Editing System for Facial Motion Capture Data. IEEE Comput. Graph. Appl. 28, 6. 76-82.

Liu, X., Mao, T., Xia, S., Yu, Y., and Wang, Z. 2008. Facial animation by optimized blend shapes from motion capture data. Comput. Animat. Virtual Worlds 19, 3-4. 235-245.

Maejima. A, Yarimizu. H, Kubo. H, and Morishima. S. 2010. Automatic generation of head models and facial animations considering personal characteristics. In Proceedings of the 17th ACM Symposium on Virtual Reality Software and Technology (VRST '10). ACM, New York, NY, USA, 71-78.

Malatesta. L, Raouzaiou. A, Karpouzis. K, and Kollias. S. 2009. MPEG-4 facial expression synthesis. Personal Ubiquitous Comput. 13, 1, 77-83. 
Manohar. V, Shreve. M, Goldgof. D, and Sarkar. S. 2010. Modeling Facial Skin Motion Properties in Video and Its Application to Matching Faces across Expressions. In Proceedings of the 2010 20th International Conference on Pattern Recognition (ICPR '10). IEEE Computer Society, Washington, DC, USA, 2122-2125.

Maraffi, C. 2003. Maya Character creation: modeling and animation control. New Riders. New York.

Orvalho, V. C. and Susin, A. 2007. Fast and reusable facial rigging and animation. In Proceedings of ACM SIGGRAPH 2007 Sketches San Diego, August 2007. 63.

Osipa, J. 2007. Stop Staring: Facial Modeling and Animation Done Right. John Wiley and Sons. New York.

Parke, F. I. 1974 A Parametric Model for Human Faces. The University of Utah. Doctoral Thesis.

Parke, F. I., AND Waters, K. 1996. Computer Facial Animation. AK Peters, Ltd. New York.

Pighin. F, Szeliski. R, and Salesin. D. 2002. Modeling and Animating Realistic Faces from Images. Int. J. Comput. Vision 50, 2,143-169.

Schmidt, F., Jagger, J., McCambell, J., and Slagel, C. 2004. 3D animation: difficult or impossible to teach and learn?. In proceedings of ACM SIGGRAPH 2004 Panels. Los Angeles, California, August 2004. J. Gibbs, Ed. SIGGRAPH '04. ACM, New York, 3-6.

Shi. F., Pei. Y, and Zha. H. 2009. Interactive modeling of 3D facial expressions with hierarchical Gaussian process latent variable models. In Proceedings of the 16th IEEE international conference on Image processing (ICIP'09), Magdy Bayoumi (Ed.). IEEE Press, Piscataway, 2449-2452.

Xia. J, He. Y, Quynh. D, Chen. X, and Hoi. S. 2010. Modeling 3D facial expressions using geometry videos. In Proceedings of the international conference on Multimedia (MM '10). ACM, New York, 591-600.

Yin. L and Wei. X. 2006. Multi-Scale Primal Feature Based Facial Expression Modeling and Identification. In Proceedings of the 7th International Conference on Automatic Face and Gesture Recognition (FGR '06). IEEE Computer Society, Washington, 603-608.

Yin. L, and Weiss. K. 2004. Generating 3D views of facial expressions from frontal face video based on topographic analysis. In Proceedings of the 12th annual ACM international conference on Multimedia (MULTIMEDIA '04). ACM, New York, 360-363.

Wang. A, Emmi. M, and Faloutsos. P. 2007. Assembling an expressive facial animation system. In Proceedings of the 2007 ACM SIGGRAPH symposium on Video games (Sandbox '07). ACM, New York, 21-26.

Waters, K. 1987. A muscle model for animation three-dimensional facial expression. In Proceedings of the 14th Annual Conference on Computer Graphics and interactive Techniques M. C. Stone, Ed. SIGGRAPH '87. California, July.17-24.

Wang. J, and Yin. L. 2007. Static topographic modeling for facial expression recognition and analysis. Comput. Vis. Image Underst. 108, 1-2, 19-34.

Waters, K., and Levergood, K. 1995. DECface: A system for synthetic face applications. Multimedia Tools and Applications. 4, 1. 349-366.

Zhang, Y., Prakash, E. C., and Sung, E. 2002. Anatomy-based 3D facial modeling for expression animation. ACM International Journal on Machine Graphics \& Vision 11, 1. 53-76.

Zhang. Y, Prakash. E, and Sung. E. 2003. Hierarchical Facial Data Modeling for Visual Expression Synthesis. J. Vis. 6, 3, 313-320. 